\title{
Curcumin and colon cancer
}

\section{Aishwarya Badiger}

Abstract: Curcumin, derived from turmeric (Curcuma longa) has been extensively studied and valued for its anti-cancer properties. It acts against a wide range of cancers by hindering the six main traits of all cancers, and the most prominent mechanisms include targeting inflammatory pathways. However, since curcumin has low bioavailability, most of it is excreted causing low systemic levels, but measurable concentrations in the colonic mucosa. Consequently, colon cancer, which has a high rate of incidence, is one of the more suited targets for curcumin action. The aim of this review is to summarize the mechanisms of curcumin in colon cancer with an emphasis on clinical studies, bioavailability and speculations about turmeric.

\section{Background}

Colorectal cancer is the third most commonly occurring cancer in the world and the fourth most common cause of cancer death ${ }^{1}$. The term colon cancer is often used synonymously with colorectal or rectal cancer because they exhibit very similar features. Most of the colon cancers begin with a mutation in the APC gene (adenomatous polyposis coli) which causes normal mucosal cells to transform into ACF (aberrant crypt foci, which are the earliest pre-malignant lesions) and eventually into adenomas ${ }^{2,3}$. Certain established risk factors for colon cancer include lifestyle factors (obesity, smoking, physical inactivity), dietary factors (low consumption of whole grains/dietary fiber, high intake of red and processed meats, alcohol consumption) and genetic factors ${ }^{1,4}$. Colon cancer is easy to detect if regular screenings are conducted. In its absence, the cancer can progress rapidly and has little chances of successful treatment. Currently used chemotherapy regimens include FOLFIRI (folinic acid, 5-fluorouracil, irinotecan) and FOLFOX (folinic acid, 5-fluorouracil, oxaliplatin) ${ }^{5}$. Due to the harsh side-effects associated with such treatments, plant-derived compounds or phytochemicals, which have little to no adverse effects, are being researched as alternatives.

Curcumin is the yellow-coloring bioactive compound present in the spice, turmeric. The first records of turmeric being used as a spice and a medicine date back to the $15^{\text {th }}$ century ${ }^{6}$. However, the discovery of curcumin is relatively recent, and it happened around 150 years back when Vogel and Pelletier isolated the yellow coloring compound in turmeric and coined the name "curcumin" 7. In the years that followed, the structure of curcumin was determined and it was synthesized artificially in $1913^{7,8}$. Further, it was discovered that curcumin is actually a mixture known as curcuminoids which make up $1-6 \%$ of turmeric ${ }^{9}$. Curcumin and its derivatives, demethoxycurcumin and bisdemethoxycurcumin are the major components of curcuminoids. In addition to curcuminoids, turmeric oil contains volatile compounds which are being studied for their biological activities. Today, at least 235 compounds in turmeric have been identified ${ }^{10}$. Preclinical studies have shown the anti-cancer activity of curcumin in different types of cancer models such as breast, gastric, head and neck, pancreatic, colon and hepatic cancers ${ }^{5}$.

Since colon cancer has such a high incidence rate, prevention through dietary pattern changes makes sense. Although lower incidences of colon cancers have been observed in the countries with higher turmeric consumptions (India and surrounding regions) ${ }^{11}$, epidemiological data on the same 
is scarce. In 2004, a Nature review identified curcumin as a potential dietary factor for colorectal cancer epidemiology study in developing countries in South and Southeast Asia ${ }^{12}$. Since Curcuma Longa is the principal rhizome consumed that contains this compound, epidemiological studies targeting turmeric consumption would be more accurate. Despite curcumin having numerous health benefits, turmeric does not appear in the 2018 WCRF/AICR colorectal cancer report ${ }^{1}$ as a dietary factor with suggestive evidence for reducing the risk of colon cancer. This is probably because most of the cancer research so far has been focused on curcumin and not turmeric. The objective of this paper is to critically review the literature on curcumin in relation to its efficacy for colon cancer prevention and treatment.

\section{Mechanisms of action: Curcumin targets the hallmarks of cancer}

Curcumin directly targets the six hallmarks of cancer as described by Hanahan and Weinberg ${ }^{13}$, leading to its versatility in the treatment of a number of different cancers. The mechanisms of curcumin action against these hallmarks in colon cancer models have been discussed below:

\subsection{Curcumin suppresses proliferative signaling}

Being a potent anti-inflammatory molecule, curcumin acts on the master regulator of

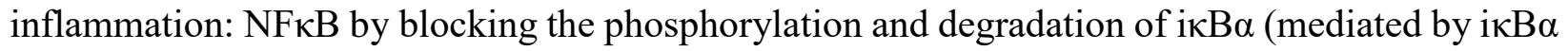

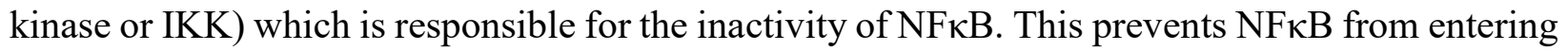
the nucleus and transcribing target genes responsible for inflammation, proliferation, angiogenesis and anti-apoptotic genes ${ }^{14}$. Curcumin also blocks the eicosanoid pathway by inhibiting COX-2 and $\mathrm{LOX}^{15}$. In CRC patients with APC gene mutations, $\beta$-catenin degradation is blocked, leading to its increased localization to the nucleus and consequently, increased expression of Wnt target genes responsible for growth and proliferation. Recently, a study ${ }^{16}$ revealed that curcumin exerts its anti-proliferative action through the suppression of the Wnt/ $\beta$-catenin pathway via miR-130a (which is overexpressed in colon cancer cells) in mice injected with colon cancer cells. Curcumin was also found to suppress CDK2 (cyclin-dependent kinase 2) activity in in vitro and ex vivo colon cancer models leading to cell-cycle arrest and reduced proliferation ${ }^{17}$.

\subsection{Curcumin induces apoptosis}

In normal cells, the tumor suppressor gene, TP53 can slow down cell-cycle progression in the presence of stress and is also capable of inducing apoptosis or programmed cell death. DNA damage triggers the expression of TP53 which transcribes the p53 protein responsible for DNA repair as a result of which the cell cycle is arrested. In case the DNA cannot be repaired, proapoptotic proteins of the Bcl2 family, such as Bax and Bak are expressed. The Bcl2 family also has anti-apoptotic proteins such as Bcl2, Bcl-xl. Curcumin decreases the expression of Bcl2 and increased $\mathrm{Bax}$ in colon cancer cells in a time-dependent manner ${ }^{18}$. In addition to this, the study also demonstrated that curcumin downregulated the activity of the anti-apoptotic protein, survivin leading to the activation of caspase- 3 and consequently cleaving PARP. The combined effect was increased apoptosis of HT-29 colon cancer cells. The curcumin derivative, bis-dehydroxy curcumin also showed similar regulation of $\mathrm{p} 53$, and the Bcl 2 family of proteins in colon cancer cells and cell death occurred through ER stress mediated autophagy ${ }^{19}$. Another study ${ }^{20}$ provided strong evidence of apoptotic activity of curcumin in CRC patients treated with the same. They 
observed an upregulation of p53, decrease in Bcl2 and an increase in Bax in colonic tissues of CRC patients treated with curcumin compared to the placebo group. Other mechanisms of apoptosis effected by curcumin include ROS production ${ }^{21}$ and inhibition of proteasome activity ${ }^{22}$ in colon cancer cells.

\subsection{Curcumin prevents angiogenesis}

As tumor cells grow, they need more vasculature to supply the required nourishment. This leads to the formation of new blood vessels around the tumor cells in a process called 'angiogenesis'. VEGF (vascular endothelial growth factor) is released by the tumor cells and goes to the receptor, VEGFR on the endothelial cells in the blood vessels near the tumor leading to a phosphorylation cascade involving PI3K/Akt, protein kinase $\mathrm{C}$ (PKC) and MAPK ultimately causing an induction of angiogenesis ${ }^{23}$. Anti-angiogenic therapies have been approved as acceptable treatments for colorectal adenocarcinoma ${ }^{24}$. It has been discovered that curcumin prevents angiogenesis in human intestinal microvascular endothelial cells stimulated with VEGF in vitro ${ }^{23}$ resulting in suppressed growth, migration and tube formation. Additionally, curcumin nano-micelles have been shown to be more effective than free curcumin in restricting angiogenesis in vitro and in vivo colon cancer models ${ }^{25}$ owing to the increased bioavailability of the nano-micelles.

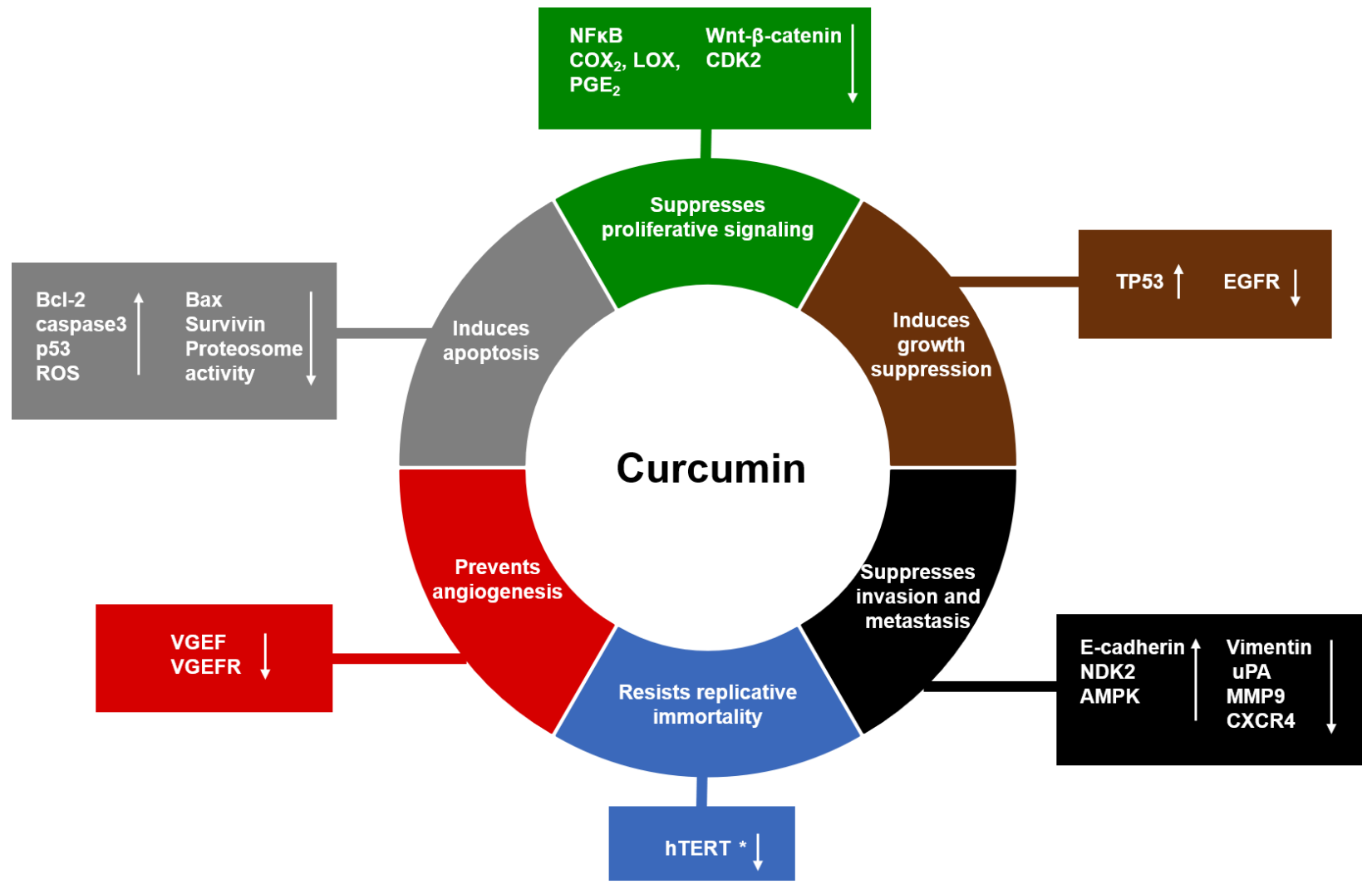

Fig. 1 Curcumin targets the classical hallmarks of cancer. The molecular targets of curcumin against colon cancer are depicted. *hTERT reduction by curcumin has been demonstrated in models other than colon cancer 


\subsection{Curcumin resists replicative immortality}

Cancer cells exhibit the maintenance of DNA telomeres causing a resistance to senescence. As a result, the cells are immortalized, and telomerase activity is high in cancer cells. Curcumin has shown inhibitory activity against human telomerase (hTERT) in a lot of cancer cell lines including breast cancer cells ${ }^{26}$, lung cancer ${ }^{27}$, leukemia ${ }^{28}$, hepatoma ${ }^{28}$, gastric cancer ${ }^{28}$ cell lines. However, to the best of our knowledge, the action of curcumin on hTERT has not been tested in colon cancer models.

\subsection{Curcumin suppresses invasion and metastasis}

Epithelial-mesenchymal transition (EMT) is one mechanism by which cancer cells gain the ability to invade and spread from the primary tumor location to other parts of the body ${ }^{13}$. Normal epithelial cells have adhesion molecules like E-cadherin, that allow the epithelial cells to maintain an organized structure. When EMT occurs, E-cadherin levels decrease with a subsequent increase in intermediate filament proteins like $\mathrm{N}$-cadherin and vimentin. Curcumin was found to inhibit EMT in human colon cancer cells by increasing the expression of E-cadherin and decreasing vimentin in addition to blocking the Wnt signaling pathway through the upregulation of NDK2, thus inhibiting proliferation ${ }^{29}$. Curcumin also suppressed metastasis of these cells by decreasing the expression of chemokine receptor 4 which is known to be important in tumor invasion and metastasis ${ }^{29}$. uPA (urokinase plasminogen activator), plasmins, cathepsins and MMPs (matrix metalloproteinases) are proteases involved in the degradation of the tumor extracellular matrix which is one of the first steps in metastasis. Curcumin is associated with the downregulation of uPA and MMP9 (target genes of NFKB) in colon cancer cells through AMPK mediated inactivation of $\mathrm{NF} \kappa \mathrm{B}$, thus blocking invasion. ${ }^{30}$

\subsection{Curcumin induces growth suppression}

The role of TP53 in tumor suppression has already been discussed earlier in this paper. In addition to TP53, cancer cells evade growth suppression by overexpressing the growth factor, EGF (epidermal growth factor) and its receptor EGFR. Curcumin decreases the expression of EGFR in human colon cancer derived cells by blocking the transcription factor Egr- ${ }^{31}$.

\section{Bioavailability issues}

One of the major downsides of curcumin, limiting its progress from in vitro and in vivo studies to clinical studies is its poor bioavailability in the human body. In a pharmacokinetic dose escalation study $^{32}$, fifteen patients with advanced colorectal cancer were orally administered with curcumin capsules containing 20mg of curcuminoids and 200mg of Curcuma essential oils. None of the curcumin or curcumin conjugates (curcumin sulphate, curcumin glucuronide, hexahydroxycurcumin and hexahydroxycurcuminol) were detected in the blood and plasma samples of any of the patients even after 29 days of everyday treatment. Not surprisingly, the feces of all fifteen patients had measurable amounts of curcumin and in the case of one patient, curcumin sulphate. In another study, after ingestion of 4 to $8 \mathrm{~g}$ of curcumin per day, only $0.016-0.0375 \%$ of it appeared in the blood and the blood serum curcumin concentration was highest at 1 to $2 \mathrm{hrs}$ of oral curcumin consumption ${ }^{33}$. In addition to being poorly absorbed, curcumin is also rapidly 
converted to its conjugates. When curcumin was consumed at higher doses (10g and $12 \mathrm{~g}$ ), curcumin conjugates (curcumin glucuronide and curcumin sulphate) were present in the blood at levels between $1-3 \mathrm{ug} / \mathrm{ml}$ with higher concentrations at the lower dosage. They have speculated that this could be due to an absorption maximum being reached as the dosage is increased leading to increased metabolism. The elevated concentrations of curcumin conjugates in the serum could also be attributed to the consumption of high-fat breakfasts by the subjects which acted as an ideal carrier for the lipophilic molecule, curcumin. ${ }^{34}$ In the past couple of decades, several drug delivery techniques are being developed to improve the absorption of curcumin without it being metabolized. These include (but are not limited to) liposomal encapsulation, nanogels, curcumin nanoparticles, inclusion complexes and nanoemulsions ${ }^{35}$.

\section{Clinical studies}

The success of curcumin in pre-clinical studies had led to seven phase I and phase II clinical trials involving colon cancer patients or subjects with a high risk of the same. In a phase I clinical trial ${ }^{36}$ involving fifteen patients with colorectal adenocarcinoma, up to $3.6 \mathrm{~g}$ of curcumin consumption per day was deemed safe. They also observed low systemic concentrations of curcumin with most of it being excreted in the feces. However, since intestinal mucosal levels of curcumin in mice were not dependent on dosage, they propose that intestinal curcumin levels may not be reflected by systemic absorption. This was validated by reports from a study ${ }^{37}$ which observed curcumin in colonic mucosa of all the patients treated with curcumin despite it not being detected in the plasma of all patients. This supports the rationale for using curcumin in the treatment of colon cancer. Although no upper limit has been established for oral consumption of curcumin, doses up to $12 \mathrm{~g}$ were well tolerated with some side effects (diarrhea, yellow stools, headache), less than grade I level toxicity or the volume of the drug being undesirable to the patients ${ }^{34,38}$. These phase I trials establish a safe dose limit that is advantageous for phase II clinical studies examining the effectiveness of curcumin. A phase IIa clinical trial ${ }^{39}$ tested the efficacy of curcumin in patients with aberrant crypt foci (ACF) by looking at ACF numbers, $\mathrm{PGE}_{2}$ and 5-HETE (as biomarkers for curcumin action) concentrations in colonic mucosa and levels of curcumin and its conjugates in plasma and mucosa. While they did not observe any significant reduction in the biomarkers, a $40 \%$ reduction in ACF numbers was seen in their $4 \mathrm{~g}$ dosage group. However, only the curcumin conjugate concentrations in plasma were significantly higher post-intervention implying one of two things: i) the curcumin conjugates seen in rectal mucosa are delivered through systemic circulation or ii) curcumin is rapidly absorbed and metabolized in the mucosa. The latter seems more plausible and is supported by evidence from other studies ${ }^{37}$ as discussed earlier. Other clinical studies involving curcumin with other phytochemicals have also been conducted.

\section{Synergistic action}

Curcumin has shown enhanced activity with a lot of other phytochemicals such as resveratrol ${ }^{40}$, piperine (alkaloid from Piper species or pepper), quercetin (plant flavanol found in onions, kale and other fruits and vegetables), epigallocatechin gallate or $\mathrm{EGCG}^{41}$ (present in green tea extract) and silymarin ${ }^{42}$ (from milk thistle seeds). One of the most well-known synergies is with piperine and it is believed to increase the bioavailability of curcumin by $2000 \%$ as compared to curcumin alone ${ }^{43}$. Another example of exceptional synergy is with quercetin where the combination 
treatment of curcumin $(1.4 \mathrm{~g})$ and quercetin $(60 \mathrm{mg})$ per day in five FAP patients reduced polyp sizes by $60.4 \%$ and polyp numbers by $50.9 \%{ }^{44}$. There is also considerable evidence that curcumin enhances the efficacy of chemotherapy for colon cancer.

\section{Would turmeric be more effective than curcumin?}

Although curcumin has been identified as the main compound with physiological benefits, there are many more compounds in turmeric that might have health benefits. In fact, there has been increasing research on the advantages of curcumin free turmeric or CFT. Studies have also suggested that turmeric oil causes curcumin to be more bioavailable and certain components of turmeric oil such as turmerine, turmerone, elemene, furanodiene, cyclocurcumin, bisacurone and germacrone have anti-inflammatory properties. ${ }^{45}$ Moreover, curcumin in natural turmeric is surrounded and possibly protected by the food matrix, causing it to be more bioavailable and less susceptible to degradation in the digestive tract. In a very interesting study ${ }^{46}$, curcumin and turmeric were dispersed in different food vehicles (corn oil, clarified butter, milk, water) and their permeability across a non-everted intestinal rat sac model was measured. Higher permeation of curcumin from turmeric was observed compared to that of pure curcumin when highly lipophilic carriers such as corn oil and clarified butter were used. They concluded that the volatile oils and other carbohydrate components present in the matrix of turmeric could be contributing to the higher permeability of curcumin from turmeric. ${ }^{46}$ Despite increasing evidence of turmeric possibly having better stability and biological activity than pure curcumin, using turmeric as a drug would be impractical due to the high volume of dosage required to achieve effective levels in the body. Nevertheless, inclusion of turmeric in everyday diets for a prolonged period might have the potential to prevent cancer.

\section{Conclusions}

There is a need for cancer treatments that are not only effective but can also preserve the quality of life of patients. Curcumin has been praised for its astounding properties and extensive potential for cancer prevention and treatment. While curcumin is believed to be the bioactive compound, the concept of using turmeric as a whole should not be overlooked. This also provides opportunities for creating functional foods by incorporating turmeric into appropriate carriers, which could possibly be a part of our daily diet. Despite compelling evidence of the biological activity of curcumin, its effectiveness seems to be limited by its poor bioavailability. Over the years, there has been a shift in curcumin research from studying its biological activities to techniques for improved bioavailability in the body. This seems to be the logical next step for curcumin to be able to transition to clinical trials and for the success of this compound as a chemopreventive agent. 


\section{References}

1. World Cancer Research Fund/ American Institute for Cancer Research. Diet, nutrition , physical activity and colorectal cancer. (2018).

2. Majumdar, A. P. N. Role of curcumin in preventing familial adenomatous polyposis. Dig Med Res 28, 180-187 (2018).

3. Patel, V. B., Misra, S., Patel, B. B. \& Majumdar, A. P. N. Colorectal Cancer: Chemopreventive Role of Curcumin and Resveratrol. Nutr Cancer. 62, 233-245 (2010).

4. American Cancer Society. Colorectal Cancer Risk Factors. (2018). Available at: https://www.cancer.org/cancer/colon-rectal-cancer/causes-risks-prevention/riskfactors.html. (Accessed: 1st December 2018)

5. Johnson, J. J. \& Mukhtar, H. Curcumin for chemoprevention of colon cancer. Cancer Lett. 255, 170-181 (2007).

6. Hutchins-wolfbrandt, A. \& Mistry, A. M. Dietary Turmeric Potentially Reduces the Risk of Cancer. Asian Pacific J. Cancer Prev. 12, 3169-3173 (2011).

7. Gupta, S. C., Patchva, S., Koh, W. \& Aggarwal, B. B. Discovery of curcumin, a component of golden spice, and its miraculous biological activities. Clin. Exp. Pharmacol. Physiol. 39, 283-299 (2012).

8. Prasad, S., Gupta, S. C., Tyagi, A. K. \& Aggarwal, B. B. Curcumin, a component of golden spice: From bedside to bench and back. Biotechnol. Adv. 32, 1053-1064 (2014).

9. Nelson, K. M. et al. The Essential Medicinal Chemistry of Curcumin. J. Med. Chem. 60, 1620-1637 (2017).

10. Li Shiyou, Yuan Wei, Deng Guangrui, Wang ping, Yang Paiying, A. B. B. Chemical composition and product quality control of turmeric ( Curcuma longa L .). Pharm. Crop. 2, 28-54 (2011).

11. Chauhan, D. Chemotherapeutic Potential of Curcumin for Colorectal Cancer. Curr. Pharm. Des. 8, 1695-1706 (2002).

12. Rastogi, T., Hildesheim, A. \& Sinha, R. Opportunities for cancer epidemiology in developing countries. Nat. Rev. Cancer 4, 909-917 (2004).

13. Hanahan, D. \& Weinberg, R. A. Hallmarks of Cancer: The Next Generation. Cell 144, P646-674 (2011).

14. Park, J. Anti-carcinogenic properties of curcumin on colorectal cancer. World J. Gastrointest. Oncol. 2, 169 (2010).

15. Rao Cv, Rivenson A, Simi B \& Reddy Bs. Chemoprevention of colon carcinogenesis by dietary curcumin, a naturally occurring plant phenolic compound. Cancer Res. 55, 259266 (1995).

16. Dou, H. et al. Curcumin suppresses the colon cancer proliferation by inhibiting Wnt/ $\beta$ catenin pathways via miR-130a. Front. Pharmacol. 8, 1-9 (2017). 
17. Lim, T. G. et al. Curcumin suppresses proliferation of colon cancer cells by targeting CDK2. Cancer Prev. Res. 7, 466-474 (2014).

18. Wang, J., Qi, L., Zheng, S. \& Wu, T. Curcumin induces apoptosis through the mitochondria-mediated apoptotic pathway in HT-29 cells. J. Zhejiang Univ. Sci. B 10, 93102 (2009).

19. Basile, V. et al. bis-Dehydroxy-Curcumin Triggers Mitochondrial-Associated Cell Death in Human Colon Cancer Cells through ER-Stress Induced Autophagy. PLoS One 8, (2013).

20. He, Z. Y. et al. Upregulation of p53 expression in patients with colorectal cancer by administration of curcumin. Cancer Invest. 29, 208-213 (2011).

21. Zhang, J. et al. Curcumin derivative WZ35 efficiently suppresses colon cancer progression through inducing ROS production and ER stress-dependent apoptosis. Am. J. Cancer Res. 7, 275-288 (2017).

22. Milacic, V. et al. Curcumin inhibits the proteasome activity in human colon cancer cells in vitro and in vivo. Cancer 68, 7283-7292 (2009).

23. Binion, D. G., Otterson, M. F. \& Rafiee, P. Curcumin inhibits VEGF-mediated angiogenesis in human intestinal microvascular endothelial cells through COX-2 and MAPK inhibition. Gut 57, 1509-1517 (2008).

24. A., P., E., C. \& J., C. New approaches in angiogenic targeting for colorectal cancer. World J. Gastroenterol. 13, 5857-5866 (2007).

25. Gao, X. et al. Improving the anti-colon cancer activity of curcumin with biodegradable nano-micelles. J. Mater. Chem. B 1, 5778-5790 (2013).

26. Ramachandran, C., Fonseca, H. B., Jhabvala, P., Escalon, E. A. \& Melnick, S. J. Curcumin inhibits telomerase activity through human telomerase reverse transcritpase in MCF-7 breast cancer cell line. Cancer Lett. 184, 1-6 (2002).

27. Lee, J. H. \& Chung, I. K. Curcumin inhibits nuclear localization of telomerase by dissociating the Hsp90 co-chaperone p23 from hTERT. Cancer Lett. 290, 76-86 (2010).

28. Cui, S.-X. et al. Curcumin inhibits telomerase activity in human cancer cell lines. Int. J. Mol. Med. 18, 227-231 (2006).

29. Zhang, Z. et al. Curcumin inhibits tumor epithelial-mesenchymal transition by downregulating the Wnt signaling pathway and upregulating NKD2 expression in colon cancer cells. Oncol. Rep. 35, 2615-2623 (2016).

30. Tong, W., Wang, Q., Sun, D. \& Suo, J. Curcumin suppresses colon cancer cell invasion via AMPK-induced inhibition of NF-кB, uPA activator and MMP9. Oncol. Lett. 12, 4139-4146 (2016).

31. Chen, A., Xu, J. \& Johnson, A. C. Curcumin inhibits human colon cancer cell growth by suppressing gene expression of epidermal growth factor receptor through reducing the activity of the transcription factor Egr-1. Oncogene 25, 278-287 (2006). 
32. Sharma, R. A. et al. Pharmacodynamic and Pharmacokinetic Study of Oral Extract in Patients with Colorectal Cancer. Clin. Cancer Res. 7, 1894 LP-1900 (2001).

33. Cheng, A.-L. et al. Phase I clinical trial of curcumin, a chemopreventive agent, in patients with high-risk or pre-malignant lesions. Anticancer Res. 21, 2895-2900 (2001).

34. Vareed, S. K. et al. Pharmacokinetics of Curcumin Conjugate Metabolites in Healthy Human Subjects. Cancer Epidemiol Biomarkers Prev. 17, 1411-1417 (2008).

35. Bose, S., Panda, A. K., Mukherjee, S. \& Sa, G. Curcumin and tumor immune-editing: Resurrecting the immune system. Cell Div. 10, 6-8 (2015).

36. Sharma, R. A. Phase I Clinical Trial of Oral Curcumin: Biomarkers of Systemic Activity and Compliance. Clin. Cancer Res. 10, 6847-6854 (2004).

37. Irving, G. R. B. et al. Prolonged biologically active colonic tissue levels of curcumin achieved after oral administration-a clinical pilot study including assessment of patient acceptability. Cancer Prev. Res. 6, 119-128 (2013).

38. Lao, C. D. et al. Dose escalation of a curcuminoid formulation. BMC Complement. Altern. Med. 6, 4-7 (2006).

39. Carroll, R. E. et al. Phase IIa clinical trial of curcumin for the prevention of colorectal neoplasia. Cancer Prev. Res. 4, 354-364 (2011).

40. Majumdar, A. P. N. et al. Curcumin synergizes with resveratrol to inhibit colon cancer. Nutr. Cancer 61, 544-553 (2009).

41. Jin, G. et al. Combination curcumin and (-)-epigallocatechin-3-gallate inhibits colorectal carcinoma microenvironment-induced angiogenesis by JAK/STAT3/IL-8 pathway. Oncogenesis 6, 1-12 (2017).

42. Montgomery, A., Adeyeni, T., San, K. K., Heuertz, R. M. \& Ezekiel, U. R. Curcumin sensitizes silymarin to exert synergistic anticancer activity in colon cancer cells. J. Cancer 7, 1250-1257 (2016).

43. Shoba, G., Joy, D., Joseph, T., Majeed Rajendran, M. R. \& Srinivas, P. Influence of Piperine on the Pharmacokinetics of Curcumin in Animals and Human Volunteers. Planta Med 64, 353-356 (1998).

44. M., C.-C. et al. Combination Treatment With Curcumin and Quercetin of Adenomas in Familial Adenomatous Polyposis. Clinical Gastroenterology and Hepatology 4, 10351038 (2006).

45. Aggarwal, B. B., Yuan, W., Li, S. \& Gupta, S. C. Curcumin-free turmeric exhibits antiinflammatory and anticancer activities: Identification of novel components of turmeric. Mol. Nutr. Food Res. 57, 1529-1542 (2013).

46. Shishu \& Maheshwari, M. Comparative bioavailability of curcumin, turmeric and Biocurcumax ${ }^{\mathrm{TM}}$ in traditional vehicles using non-everted rat intestinal sac model. J. Funct. Foods 2, 60-65 (2010). 